\title{
Self-Assembly of Facially Amphiphilic Dendrimers on Surfaces
}

\author{
Yangbin Chen, Ashootosh V. Ambade, Dharma Rao Vutukuri, and S. Thayumanavan * \\ Department of chemistry, University of Massachusetts, Amherst, Massachusetts 01003
}

\begin{abstract}
Facially amphiphilic dendrimers have been shown to provide significant difference in surface behavior due to subtle changes in structure. The monodendrons are capable of providing hydrophobic surfaces, while the didendrons provide superhydrophobic surfaces. This provides an example of how a molecular level change could result in significant changes in surface behavior. This difference is attributed to the conformational differences exhibited by these dendrimers on surfaces.
\end{abstract}

Amphiphilic dendrimers have attracted tremendous interest for both fundamental and applied research, since these molecules have the potential to form well-controlled and stable selfassembled structures. ${ }^{1}$ On solid surfaces, these molecules have been of interest for a variety of reasons, including in applications such as sensors. ${ }^{2}$ The amphiphilicity in these molecules is brought about by the difference in polarity of the focal point of the dendron (or core of a dendrimer) $v s$. the peripheral functionalities. We have recently reported on a new class of amphiphilic dendrimers, in which every repeat unit within the macromolecule contains hydrophilic and hydrophobic functionalities on opposite faces of dendritic backbone. ${ }^{3}$ These dendrimers have been shown to form micelle-type or inverse micelle-type assemblies, depending on the solvent environment. ${ }^{4}$ Considering these are rather unique self-assemblies, it is interesting to ask: what would be the nature of the self-assembled structures obtained from these facially amphiphilic dendrimers, when presented to two-dimensional surfaces? This question is interesting because, while the solution self-assembly of an amphiphile is driven by the need to minimize the interaction between the incompatible functionalities in the amphiphile with the three-dimensional surface of the bulk solvent, interaction with the solid surface is twodimensional. Would the interaction be such that these facially amphiphilic dendrimers could modify polar surfaces to apolar surfaces and vice versa? How does the surface behavior of these dendrimers differ from the corresponding polymers? Also, note that investigating both solution and solid-surface assemblies could provide information on the limiting conformers that these dendrimers are capable of adapting. Upon investigating the molecules 1-7 (Chart 1) on solid surfaces, we were surprised to realize how sensitive the surface assemblies are to structure of the dendrimer. We disclose these findings in this communication.

DMF was chosen as solvent for coating the silica surfaces, since the dendrimers do not preaggregate in DMF, as determined by dynamic light scattering. ${ }^{5}$ The modified surfaces were studied using dynamic water contact angle (CA) measurements, where both advancing $\left(\theta_{\mathrm{a}}\right)$ and receding $\left(\theta_{\mathrm{r}}\right)$ CAs are measured. ${ }^{6}$ The $\operatorname{CAs}\left(\theta_{\mathrm{a}} / \theta_{\mathrm{r}}\right)$ for the silica itself was determined to be $18^{\circ} / 8^{\circ}$. Upon coating the small molecule model compound 1 , the CAs changed to $65^{\circ} / 9^{\circ}$. Although there is a large change in the advancing angle, the receding angle is essentially unchanged resulting in a big hysteresis. When the first generation dendron 2 was used, one could already notice the dendritic effect as the CAs increased to $91^{\circ} / 70^{\circ}$. The CAs observed for dendrons 3 and 4 are $92^{\circ} / 73^{\circ}$, and $94^{\circ} / 77^{\circ}$, respectively (Figure 1). Note that the normal dendritic effects are observed at higher generations in solution. ${ }^{4}$ On these surfaces however, 
the enhancement due to the dendritic architecture is observed even at very low generations. One could argue that this is simply a polyvalency effect between the carboxylic acid of the dendrons and hydroxy groups on the silica surface. We suggest however that this is a dendritic effect, since a similar polymer $8\left(\mathrm{M}_{\mathrm{n}}=17225\right.$, PDI=1.44) with similar functionalities did not display such high CAs under these conditions without heating (vide infra).

The most surprising results were obtained, when we measured the CAs for the didendrons 57. The first generation didendron, $\mathbf{5}$, exhibited much higher CAs of $120^{\circ} / 95^{\circ}$ (Figure 1). These angles increased further to $148^{\circ} / 131^{\circ}$ and $140^{\circ} / 126^{\circ}$, when the second and third generation didendrons ( 6 and 7 ) were used. These latter values are typical for superhydrophobic surfaces. A superhydrophobic surface is obtained, when a hydrophobic surface is rendered rough, which is typically achieved by various processing techniques. ${ }^{7}$ To our knowledge, there is no prior report on hydrophobic to superhydrophobic transition solely through architectural control at a molecular level. Note that this change is not a simple manifestation of increasing molecular weights. For example, the molecular weights of $4(\mathrm{MW}=5577.14)$ and $\mathbf{6}(\mathrm{MW}=5359.02)$ are nearly the same. This suggests that subtle differences in didendron $v s$. monodendron architecture play a crucial role in the surface behavior.

In all cases, carboxylic acid units are directed towards the polar silica surface and alkyl groups are displayed on the top of the surface. The driving force for such a presentation is two-fold: (i) the carboxylic acids benefit from hydrogen bonding interactions with the hydroxy groups of the silica surface. (ii) The alkyl groups have lower surface energy compared to carboxylic acid groups and therefore prefer to be displayed at the solid-air interface. ${ }^{8} \mathrm{We}$ propose the following hypothetical model to explain the differences. The monodendrons are capable of exhibiting a conformation, where all carboxylic acid units within the dendrons are capable of being in contact with the polar surface of silica (Figure 2a). However, in the case of didendrons, two monodendrons are brought in close proximity by a bisphenol-A moiety. It is possible that this feature does not allow for a conformation in which all carboxylic acids can be in contact with the surface. Since the periphery has the maximum number of carboxylic acid units, these units are in contact with the surface compared to the core of the dendrimer, as schematically illustrated in Figure 2b. This conformational difference could be responsible for the surface roughness observed with the didendrons. A key difference is that the dendrons $\mathbf{2 - 4}$ have a hydroxyl moiety at the focal point, whereas the dendrimers 5-7 do not. We have converted the $-\mathrm{OH}$ group of dendron $\mathbf{3}$ to a phenyl ether, the surface behavior of which is similar to $\mathbf{3}$ itself. 5 This suggests that the $-\mathrm{OH}$ group does not play a significant role.

To test the hypotheses, we carried out the following experiments. First, we needed to test whether our dendrimers form just monolayers. From ellipsometry, ${ }^{5}$ layer thickness with monodendrons 2-4 was about 20-23 $\AA$ and was about 22, 30, and $35 \AA$ for didendrons 5-7 respectively. The end-to-end distance between carboxylic acid moiety and hydrophobic decyl moiety was estimated to be about $18 \AA$. Therefore, the numbers above suggest that a monolayer is obtained with dendrons $\mathbf{2 - 4}$. The increase in the layer thickness for $\mathbf{6}$ and $\mathbf{7}$ was taken to be consistent with the curvature produced by these dendrimers (schematically represented in Figure $2 b$ ). Note however that ellipsometry on a rough surface merely provided an estimate that it is close to a monolayer each of the detected spot. Also, if the surface roughness is indeed different for the mono- $v s$. didendrons, it is possible that atomic force microscopy (AFM) provides the evidence for the differences. AFM height images of dendron 4 and dendrimer 6 are shown in Figure $2 \mathrm{c}$ and $2 \mathrm{~d} .{ }^{5}$ Dendrons seem to form aggregated disc type structures on the surfaces. Note that the diameters of the seemingly spherical assemblies are about $200 \mathrm{~nm}$, whereas the heights are much lesser than $4 \mathrm{~nm}$. On the other hand, the surfaces formed by didendrons are much less aggregated and rougher. The AFM images of the didendrons are reminiscent of those obtained for lotus leaves. ${ }^{9} \mathrm{We}$ also utilized AFM to identify whether there 
is any crystallization of the alkyl chains in either of these architectures and found no such evidence. $^{5}$

An interesting question to ask is: are these kinetically formed supramolecular assemblies or do these represent thermodynamic minima? Thermodynamically driven assemblies have the advantage that they are less likely to be perturbed by temporary environmental stress. To address this, we heated these dendrimercoated surfaces for a few hours above the $T_{g}$ of the dendrimers. ${ }^{5}$ We observed that the contact angles for the surfaces exhibited no significant change. This result was taken to suggest that the assemblies obtained are indeed the result of the assembly finding a thermodynamic minimum during the coating process.

Are dendrimers unique in their capability or could one achieve superhydrophobic surfaces with a structurally similar polymer? We utilized facially amphiphilic polymer $\mathbf{8}$, which exhibit micelle/inverse micelle properties similar to those of the dendrimers, ${ }^{10}$ to investigate the surface behavior. CAs of $56^{\circ} / 38^{\circ}$ were obtained, and after this surface was heated above the $\mathrm{T}_{\mathrm{g}}$ of the polymer 8 , CAs changed to $95^{\circ} / 45^{\circ}$. These results show that the polymers $(i)$ could not provide superhydrophobic surfaces; (ii) a kinetically driven conformation is first achieved upon coating the surfaces, in the case of polymers.

Finally, we were interested in finding whether these dendrimers are capable of converting hydrophobic surfaces to hydrophilic surfaces. When a hydrophobized silica surface with CAs of $102^{\circ} / 100^{\circ}$ was used as the substrate for coating the dendrimers, very little change in the CAs was observed. ${ }^{5}$ This is understandable, since in this case the lower surface energy of hydrophobic alkyl chains and hence the tendency to be presented at solid-air interface and the surface preference for hydrophobic alkyl groups work against each other. To compensate for this, these dendrimers could be forming a bilayer or a multilayer. Indeed, the surfaces were found to be uneven with layer thicknesses ranging from 40 to $70 \AA$.

In summary, we have shown that: (i) the facially amphiphilic dendrimers exhibit a dendritic effect in surface modifications. (ii) While the monodendrons provide hydrophobic surfaces, the didendrons provide superhydrophobic surfaces. This is attributed to the possible conformational differences between these architectures on the surface. (iii) These dendrimers provide thermodynamically driven assemblies; the corresponding polymers do not. (iv) Hydrophobic surfaces could not be converted to hydrophilic surfaces by these dendrimers, due to surface affinity $v s$. surface energy competition of alkyl groups. Further theoretical and experimental studies are needed to understand the molecular level control over surface modifications.

\section{Supplementary Material}

Refer to Web version on PubMed Central for supplementary material.

\section{Acknowledgements}

We thank the NIGMS of the NIH (GM-65255) for support and NSF-MRSEC for infrastructure support.

\section{References}

1. Newkome, GR.; Moorefield, CN.; Vogtle, F. Dendrimers and Dendrons: Concepts, Syntheses, Applications. Wiley-VCH; New York: 2001. (b) Sayed-Sweet Y, Hedstrand DM, Spinder R, Tomalia DA. J Mater Chem 1997;7:1199. (c) Baars MWPL, Kleppinger R, Koch MHJ, Yeu SL, Meijer EW. Angew Chem, Int Ed 2000;39:1285. (d) Hawker CJ, Wooley KL, Fréchet JMJ. J Chem Soc, Perkin Trans 1 1993;12:1287. (e) Newkome GR, Moorefield CN, Baker GR, Saunders MJ, Grossman SH. Angew Chem, Int Ed 1991;30:1178. (f) Klaikherd A, Sandanaraj BS, Vutukuri D, Thayumanavan S. J Am Chem Soc 2006;127:9231. [PubMed: 16834397] and references therein 
2. (a) Tully DC, Frechet JMJ. Chem Commun 2001:1229. (b) Crooks RM, Ricco AJ. Acc Chem Res 1998;31:219. (c) Xiao Z, Cai C, Mayeux A, Milenkovic A. Langmuir 2002;18:7728.and references therein

3. Bharathi P, Zhao H, Thayumanavan S. Org Lett 2001;3:1961. [PubMed: 11405755]

4. Vutukuri DR, Basu S, Thayumanavan S. J Am Chem Soc 2004;126:15636. [PubMed: 15571373]

5. See Supporting Information for details.

6. Oner D, McCarthy TJ. Langmuir 2000;16:7777.

7. Jiang L, Zhao Y, Zhai J. Angew Chem, Int Ed 2004;43:4338. and references therein

8. Ulman, A. An Introduction to Ultrathin Organic Films: From Langmuir-Blodgett to Self-Assembly. Academic Press; London: 1991.

9. University of Bath. [(accessed Oct 2006)]. http://people.bath.ac.uk/en2ral/lotussurface.html

10. (a) Basu S, Vutukuri DR, Shyamroy S, Sandanaraj BS, Thayumanavan S. J Am Chem Soc 2004;126:9890. [PubMed: 15303841] (b) Basu S, Vutukuri DR, Thayumanavan S. J Am Chem Soc 2005;127:16794. [PubMed: 16316219] 


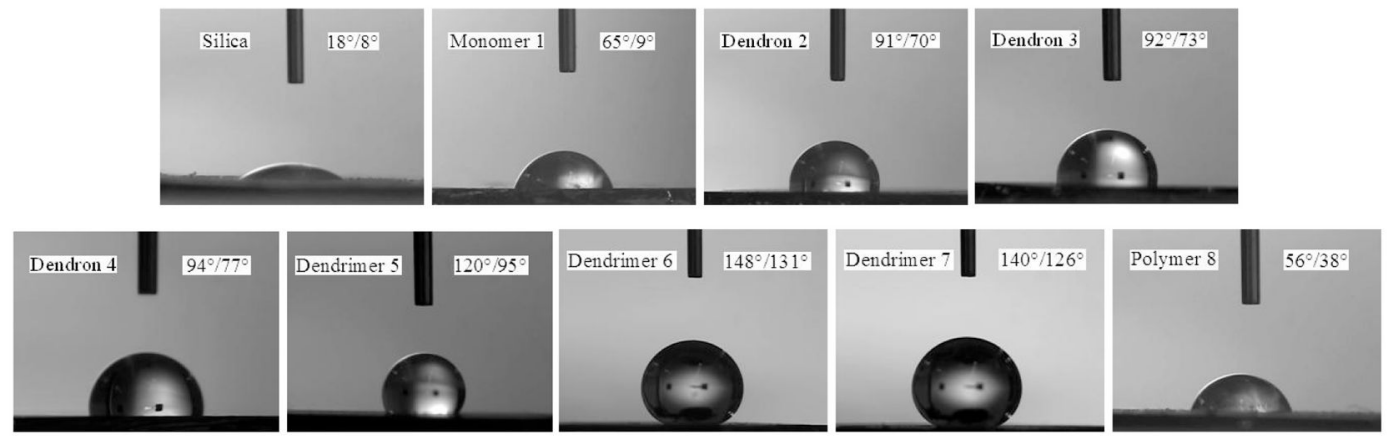

Figure 1.

CAs $\left(\theta_{a} / \theta_{r}\right)$ and droplet profiles of compounds 1-8. 

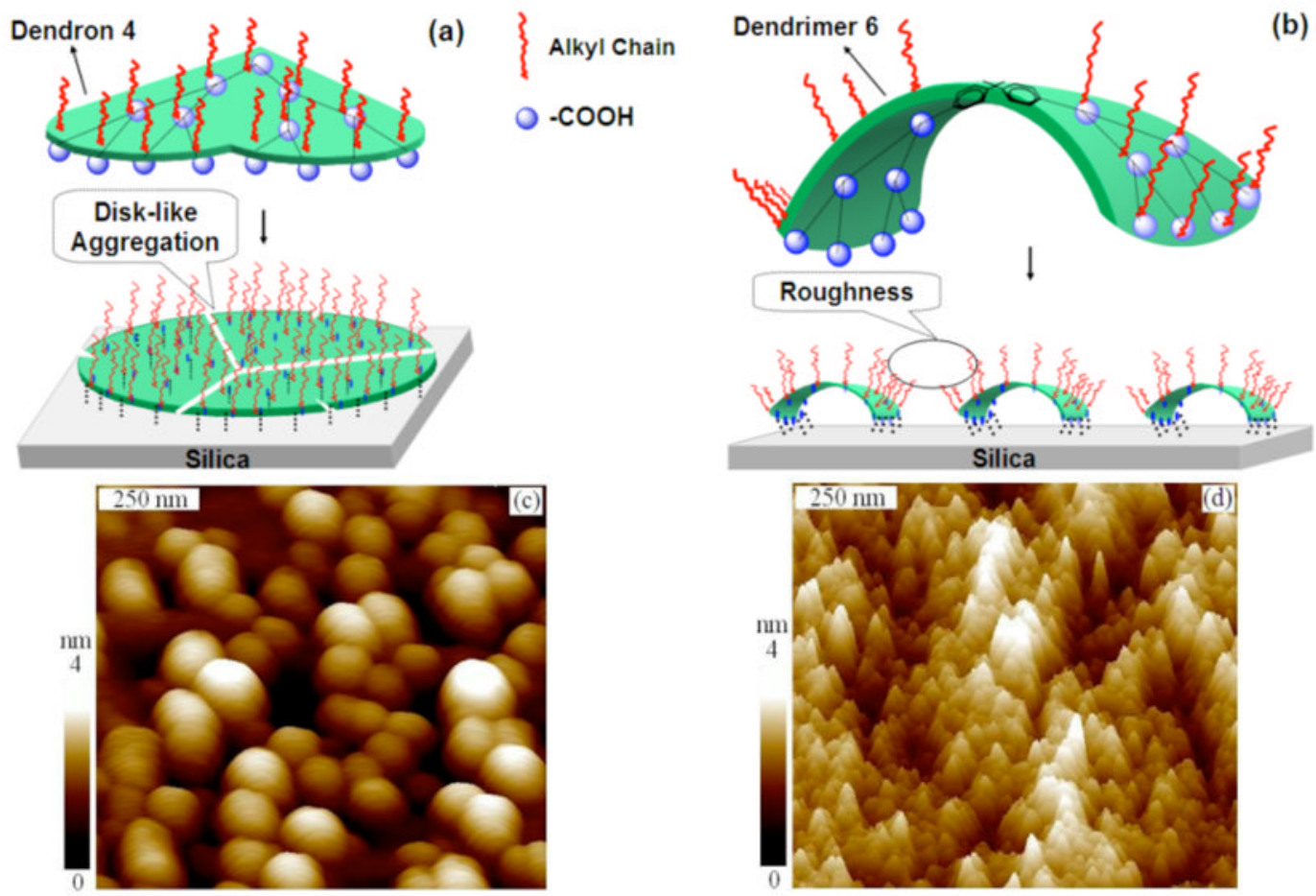

Figure 2.

Hypothetical conformational schematic for (a) dendron;(b) dendrimers. AFM height images of (c) 4 ; (d) 6 . 

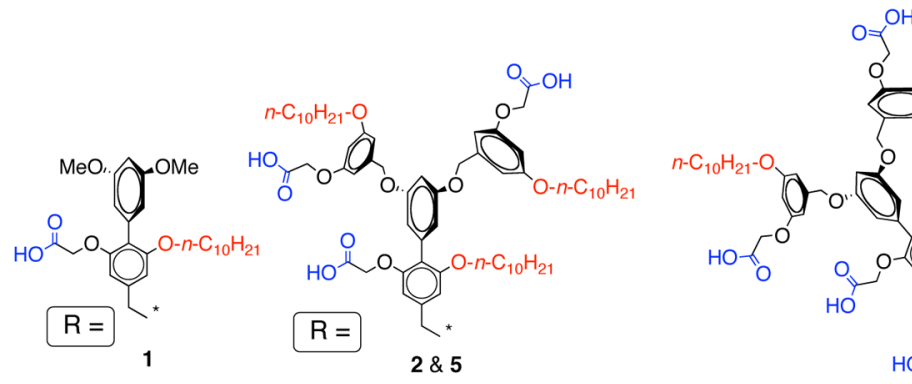

$$
\gamma^{\mathrm{OH}}
$$
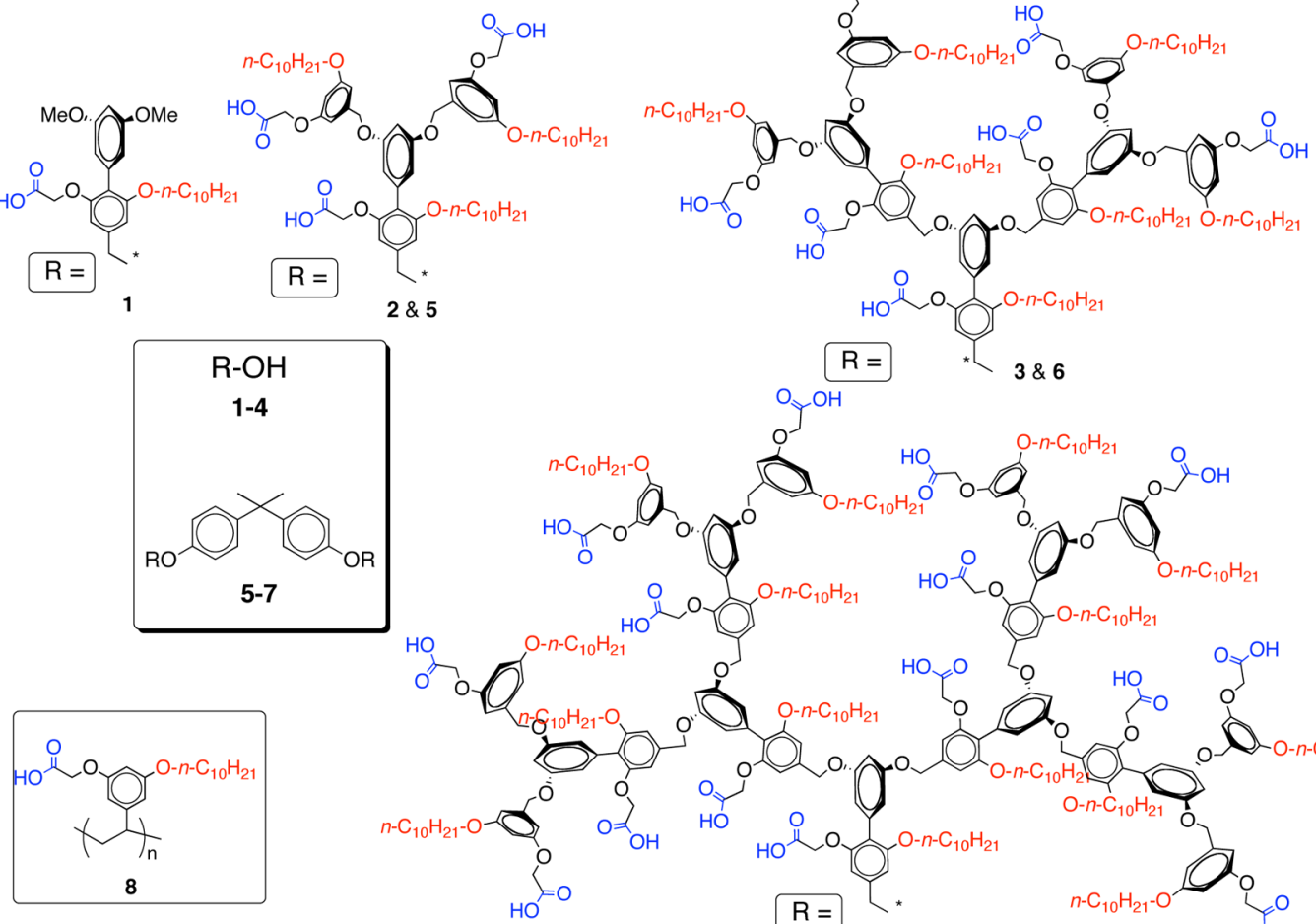

Chart 1.

Structures of amphiphilic dendrimers and polymer. ${ }^{5}$ 